\title{
Interference Alignment with Frequency-Clustering for Efficient Resource Allocation in Cognitive Radio Networks
}

\author{
Mohammed El-Absi, Musbah Shaat, Faouzi Bader, and Thomas Kaiser
}

\begin{abstract}
In this paper, we investigate the resource management problem in orthogonal frequency division multiplexing (OFDM) based multiple-input multiple-output (MIMO) cognitive radio (CR) systems. We propose to perform resource allocation based on interference alignment (IA) in order to improve the spectral efficiency of CR systems without affecting the quality of service of the primary system. IA plays a role in the proposed algorithm to enable the secondary users (SUs) to cooperate and share the available spectrum, which leads to a considerable increase in the spectral efficiency of CR systems. However, IA based spectrum sharing is restricted to a certain number of SUs per subcarrier in order to satisfy the IA feasibility conditions. Accordingly, the resource allocation problem is formulated as a mixed-integer optimization problem which is considered as an $\mathcal{N} \mathcal{P}$-hard problem. To reduce the computational complexity of the problem, a two-phases efficient sub-optimal algorithm is proposed. In the first phase, frequency-clustering is performed in order to satisfy the IA feasibility conditions, where each subcarrier is assigned to a feasible number of SUs. Whenever possible, frequency-clustering stage considers the fairness among the SUs. In the second stage, the available power is allocated among the subcarriers and SUs without violating the constraints that limit the maximum interference induced to the primary system. Simulation results show that IA with frequency-clustering achieves a significant sum rate increase compared to CR systems with orthogonal multiple access transmission techniques.
\end{abstract}

Index Terms

Cognitive radio, Interference alignment, Frequency-clustering, Multicarrier, OFDM, MIMO, Resource Allocation.

\section{INTRODUCTION}

The governmental agencies are currently regulating the frequency allocation with a static spectrum licensing model. By this model, the spectrum is divided into several bands that are generally allocated exclusively to specific users or services. As conducted by practical measurements [2], this model leads to inefficient use of the spectrum which opposes the increasing demand of the frequency spectrum and, hence, the rapid growth of the communication services. Cognitive radio (CR) is proposed to overcome the spectrum underutilization problem by introducing a new licensing scheme which allows a group of users called secondary users (SUs) to access the vacant portion of the spectrum left by the licensed users, also called primary users (PUs), without affecting the performance of the licensed system or inducing harmful interference to it.

Multicarrier transmission schemes like orthogonal frequency division multiplexing (OFDM) offer several advantages over the single carrier ones in CR context. Multicarrier schemes provide high spectral efficiency and robustness to selective fading. Additionally, they offer more flexibility in distributing the system resources among the different users and subcarriers. Furthermore, multicarrier systems have the ability

M. El-Absi and T. Kaiser are with the Institute of Digital Signal Processing, Duisburg-Essen University, 47057 Duisburg, Germany (e-mail: mohammed.el-absi@uni-due.de).

M. Shaat is with Centre Tecnolgic de Telecomunicacions de Catalunya (CTTC), 08860 Barcelona, Spain.

F. Bader is with Suplec, 35576 Cesson-Sevigne, France.

Manuscript received October 24, 2014; revised March 13, 2015 and June 5, 2015; accepted July 26, 2015. Part of this work was supported by the Spanish Government under project ELISA (TEC2014-59255-C3-1-R).Part of this work has been accepted to be presented in IEEE GLOBECOM 2014 [1]. 
to operate in noncontiguous portions of the spectrum and have the capability to control the transmission parameters to avoid inducing severe interference to the PUs, which make it very attractive for the CR applications. Combining multiple-input multiple-output (MIMO) technology with multicarrier transmission can increase the diversity gain and accordingly the system data rate. The MIMO multicarrier systems have been considered recently as a promising candidate for CR systems.

The resource management problem in non-cognitive and cognitive systems is widely considered in the literature [3]-[9]. In the works of [3], [4] and references therein, resource management algorithms were proposed for the non-cognitive scenarios which is not always efficient within the CR context. This is because the limitation introduced by interference constraint to the CR system should be taken into consideration. Optimum power allocation with beamforming is performed to maximize the capacity without violating the interference and power constraints in [5], [6]. A game theory based decentralized approach is proposed in [7] to design a cognitive MIMO transceivers. In [8], the capacity provided by the MIMO is utilized to construct a cooperative paradigm that can be applied by the SUs to simultaneously relay the PUs traffic and transmit their own traffic over the same accessed band. Nguyen and Krunz in [9] translated the non-convex resource allocation optimization problem into a distributed non-cooperative game. At each of the CR nodes, precoding matrices are computed to maximize the capacity while preventing the interference between PUs and other SUs.

Interference alignment (IA) is a cooperative interference management technique that reduces the dimensionality of the interference subspace aiming at maximizing the degrees-of-freedom (DoF) of $K$-user interference channels [10]. The basic concept of IA in MIMO systems is to design linear precoders at the transmitter sides and interference suppression decoders at the receiver sides in a smart way in order to align the interference signals in half of the spatial subspaces at the receiver sides.

Recently, IA has been investigated in MIMO CR systems with full cooperation between the PUs and SUs in order to allow the SUs to utilize both free and non-free eigenmodes of PUs. This employment helps in removing the interference constraints from the optimization problem since it assumes that the PUs cooperate with the SUs and can suppress the received interference at the primary side [11]-[15]. In [11], the authors considered only one MIMO SU link to coexist with one MIMO PU link aiming at that the SU achieves the same transmission rate as of the PU. This work was extended in [12] by redesigning the decoding matrix of the SU receiver in order to combat PU interference in a more effective manner, where the SU is enabled to compute blindly the required channel state information (CSI). Similarly, the work in [13] enables one SU to share the unused eigenmodes of the PU considering the power and interference constraints. In the same way, the work in [14] considered MIMO employment at SUs and a PU with frequency scheduling. In [15], the authors formulated the cooperative spectrum leasing with IA into a Stackelberg game, where the PU is the leader, and SUs are followers. This work assumed feasible IA system, which is not always valid. In [16], a limited number of SUs are enabled to share the non active primary bands based on IA in order to coexist with a single antenna PUs. Additionally, the impact of the physical layer modulation technique is studied in [17], [18]. Resource management in IA is not sufficiently addressed in the literature. The works in [19] and [20] proposed an energyefficient IA algorithm through power allocation and transmission mode adaptation for green IA systems in non-cognitive and cognitive scenarios, respectively. The aforementioned works assumed the existence of a certain level of coordination and cooperation between cognitive and primary systems. Nevertheless, the cooperation between the primary and cognitive systems is not always guaranteed, and it requires a permission from the primary system to denote some of its DoF to the SUs. Furthermore, to the best of the authors' knowledge, overloaded CR networks -where IA problems are infeasible- have not been considered in the literature. Additionally, because of the challenges associated with joint power and spectrum optimization, most existing works on MIMO IA CR systems do not consider resource management over the multicarrier systems (frequency dimension) as it is not fairly trivial.

In this paper, IA with frequency-clustering is proposed in overloaded CR systems in order to improve the spectral efficiency of MIMO CR systems while protecting the primary system performance. The tackled system model considers a practical scenario by assuming that there is no coordination between the 
cognitive and the primary network. IA based resource management problem in CR systems is formulated, where the using of IA increases the DoF per SU by enabling the SUs to effectively share the available spectrum. In the problem formulation, each subcarrier is assigned to a feasible number of SUs in order to meet the IA feasibility conditions, where the fair distribution of the resources among the different SUs is taken into account. Considering that there is no coordination between the primary and the cognitive systems, the primary system should be protected from receiving severe induced interference from the CR systems by ensuring that the received interference is below a prescribed limit. Accordingly, several interference constraints are added to the optimization problem. As the computational complexity of the optimal scheme is quite high, the paper further proposes an efficient sub-optimal resource allocation algorithm with two phases. In the first phase, frequency-clustering method is employed in order to assign each subcarrier to a feasible number of SUs with fairness consideration. Frequency-clustering operation considers the interference channel qualities of the subcarriers as well as the generated interference to PUs. In the second phase, the power is allocated among all subcarriers and SUs considering the power budget of the SUs and the interference limits at the PUs.

In this context, a preliminary investigation to this problem is addressed in [1]. However, the work of [1] suffers from a remarkable performance loss in the low interference constraints regime since the frequency-clustering is performed considering only the channel qualities, where the subcarriers with high interference gains will potentially have a low transmitting power even when they have a good channel quality. In [1], the fairness between the SUs is not considered through frequency clustering, which leads to poor sum rate for some SUs. This occurs because the subcarriers allocated to those SUs are not enough to achieve the minimum required instantaneous data rate. Motivated by these drawbacks, we propose in this work a frequency-clustering algorithm that considers not only the channel qualities and per-user power budget constraints but also takes into account the induced interference to the PU band, which achieves a significant sum rate gain in comparison with the work in [1]. Moreover, the proposed frequency-clustering phase takes the fairness among SUs into consideration. Furthermore, the work in this paper is generalized to consider the multiple antennas primary systems in addition to the case of having a multi-streams CR system.

The rest of this paper is organized as follows. The system model is described and the optimization problem is formulated in Section II. Section III presents the frequency-clustering phase with fairness consideration. The optimal and the low-complexity power allocation algorithms are introduced in Section IV. The complexity analysis is presented in Section V. Section VI exhibits and discuses the simulation results. Finally, Section VII concludes the paper.

\section{System Model and Problem Formulation}

In this work, a secondary communication system with $K$ SUs is considered, where each SU has one transmitter with $M_{T}$ antennas in order to communicate with one receiver with $M_{R}$ antennas. The assumed secondary system is co-located with a primary system in the same geographical area. The PUs are assumed to be equipped with $M_{P}$ antennas. The side-by-side frequency distribution of active and non-active bands is assumed as shown in Fig. 1. The active primary system bands represent the portions of the spectrum already occupied by the PUs while the non-active bands refer to the vacant bands that can be used by SUs. $L$ active PU bands $\left(W_{1}, W_{2}, \ldots, W_{L}\right)$ are assumed. Additionally, the non-active bands are divided into $N$ equal subcarriers each with $\Delta f$ bandwidth. The SUs are connected to a local gateway, which works as a centralized controller and is in charge of the resource management task of the network. Fig.2 shows an example of 6 SUs, in which the transmission of the different SUs causes interference to the PUs as well as to the other unintended SU receivers. The induced interference should not exceed the prescribed limit of the allowable interference that can be tolerated by each PU, i.e. $I_{t h}^{l}$. The numbers above the arrows represent the frequency-clustering that will be described later in Section III.

In our model, the transmission on a given subcarrier is not restricted to one user at a given time. Rather, different SUs are allowed to share the different subcarriers by employing IA. Accordingly, the interference 


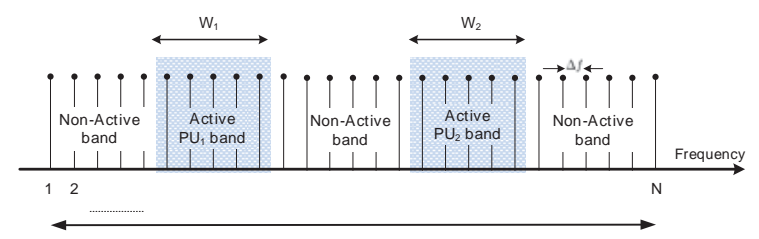

Fig. 1: Frequency distribution of active and non-active bands.

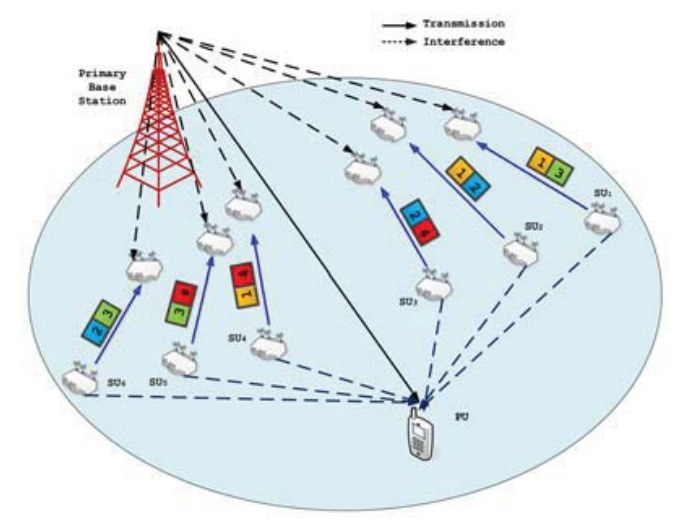

Fig. 2: Example of a CR network with 6 SU pairs. Different numbers and colors denote different subcarriers.

between SUs is managed by generating different precoding matrices based on MIMO IA technique [21], [22]. By considering a multicarrier technique, the frequency orthogonality can be achieved between subcarriers, which enables the independent application of IA on each subcarrier. Each SU transmitter sends $d$ data streams to its intended receiver. The transmitted data stream $\mathbf{x}_{k}^{n} \in \mathbb{C}^{d \times 1}$ over the $n^{\text {th }}$ subcarrier is multiplied by the precoding matrix $\mathbf{V}_{k}^{n} \in \mathbb{C}^{M_{T} \times d}$. Using this precoding, the desired data is aligned at its own receiver in the interference-free subspace while the interference signals from other SU transmitters are aligned at the interference subspace [10], [23]. By assuming a perfect knowledge of the CSI at each node, the discrete-time complex received signal at the $k^{\text {th }}$ receiver over the $n^{\text {th }}$ subcarrier is represented as

$$
\mathbf{y}_{k}^{n}=\mathbf{U}_{k}^{n \mathrm{H}} \mathbf{H}_{k k}^{n} \mathbf{V}_{k}^{n} \mathbf{x}_{k}^{n}+\sum_{j=1, j \neq k}^{K} \mathbf{U}_{k}^{n \mathrm{H}} \mathbf{H}_{k j}^{n} \mathbf{V}_{j}^{n} \mathbf{x}_{j}^{n}+\mathbf{U}_{k}^{n \mathrm{H}} \mathbf{z}_{k}^{n},
$$

where $\mathbf{U}_{k}^{n} \in \mathbb{C}^{M_{R} \times d}$ is an orthonormal linear interference suppression matrix applied at the $k^{\text {th }} \mathrm{SU}$ receiver, $\mathbf{H}_{k j}^{n} \in \mathbb{C}^{M_{R} \times M_{T}}$ denotes the channel frequency response between $j^{\text {th }}$ SU transmitter and $k^{\text {th }} \mathrm{SU}$ receiver, and $\mathbf{z}_{k}^{n} \in \mathbb{C}^{M_{R} \times 1}$ is the zero mean unit variance circularly symmetric additive white Gaussian noise (AWGN) vector at the $k^{\text {th }}$ SU receiver.

If perfect IA is achieved, only the undesired SU interfering signals to the $k^{\text {th }}$ SU receiver is aligned and cancelled. That is [10]

$$
\operatorname{rank}\left(\mathbf{U}_{k}^{n \mathrm{H}} \mathbf{H}_{k k}^{n} \mathbf{V}_{n}^{n}\right)=d \quad \forall k \quad \text { and } \quad \forall n
$$


and

$$
\mathbf{U}_{k}^{n \mathrm{H}} \mathbf{H}_{k j}^{n} \mathbf{V}_{j}^{n}=0 \quad \forall j \neq k \quad \text { and } \quad \forall n .
$$

It was stated in [24], [25] that MIMO IA in $\left(M_{R} \times M_{T}, d\right)^{K}$ interference channels is feasible when

$$
M_{T}+M_{R}-(K+1) d \geq 0 .
$$

The interference can be completely eliminated at each SU receiver when the feasibility condition in (4) is achieved. Furthermore, the precoding and decoding matrices can be designed to achieve perfect IA using closed-form solution or other algorithmic methods as presented in the literature for many cases (e.g. [10], [26]-[30] and references therein). Assuming perfect IA is achieved, the received signal in (1) becomes

$$
\mathbf{y}_{k}^{n}=\mathbf{U}_{k}^{n \mathrm{H}} \mathbf{H}_{k k}^{n} \mathbf{V}_{k}^{n} \mathbf{x}_{k}^{n}+\mathbf{U}_{k}^{n \mathrm{H}} \mathbf{z}_{k}^{n}
$$

and, hence, the total sum rate of the SUs over the $n^{\text {th }}$ subcarrier is

$$
R_{T}^{n}=\sum_{k=1}^{K} R_{k}^{n}\left(\mathbf{H}_{k k}^{n}, S_{k}^{n}\right),
$$

where $R_{k}^{n}$ is the capacity of the $k^{t h}$ SU over the $n^{t h}$ subcarrier and can be expressed as

$$
R_{k}^{n}\left(\mathbf{H}_{k k}^{n}, \mathbf{S}_{k}^{n}\right)=\log \left|\mathbf{I}_{d}+\frac{1}{\sigma_{\text {AWGN }}^{2}+\sum_{l=1}^{L} J_{l, k}^{n}} \mathbf{U}_{k}^{n \mathrm{H}} \mathbf{H}_{k k}^{n} \mathbf{V}_{k}^{n} \mathbf{S}_{k}^{n} \mathbf{V}_{k}^{n \mathrm{H}} \mathbf{H}_{k k}^{n}{ }^{\mathrm{H}} \mathbf{U}_{k}^{n}\right| .
$$

$\mathbf{S}_{k}^{n}=\mathbb{E}\left[\mathbf{x}_{k}^{n} \mathbf{x}_{k}^{n \mathrm{H}}\right] \in \mathbb{R}^{d \times d}$ is the input covariance matrix of the $k^{\text {th }}$ SU at the $n^{\text {th }}$ subcarrier and can be expressed as $\mathbf{S}_{k}^{n}=\operatorname{diag}\left(P_{k, n}(1), . ., P_{k, n}(d)\right)$, where $P_{k, n}(i)$ is the allocated power to the $i^{t h}$ data stream at the $k^{\text {th }}$ SU over the $n^{\text {th }}$ subcarrier. Therefore, the transmitted power by the $k^{\text {th }}$ SU over the $n^{\text {th }}$ subcarrier is $P_{k, n}=\operatorname{Tr}\left(\mathbf{S}_{k}^{n}\right)$. Moreover, $J_{k l}^{n}$ is the total interference introduced by the $l^{\text {th }}$ PU transmitter at the $n^{t h}$ subcarrier to the $k^{\text {th }} \mathrm{SU}$ [6], which can be expressed as

$$
J_{k l}^{n}(D n)=\sum_{m=1}^{M_{R}} \sum_{i=1}^{M_{P}}\left(\int_{D n-\Delta f / 2}^{D n+\Delta f / 2}\left|y_{k l}^{n, m, i}\right|^{2} \psi_{l}\left(e^{j \omega}\right) d \omega\right)
$$

where $D n$ represents the spectral distance between the $n^{\text {th }} \mathrm{CR}$ subcarrier and $l^{\text {th }}$ PU band. $\psi_{l}\left(e^{j \omega}\right)$ is the power spectral density (PSD) of the $l^{t h}$ PU signal, and $y_{k l}^{n, m, i}$ is the channel gain between the $m^{t h}$ SU antenna at the $k^{t h}$ SU receiver and the $i^{t h}$ antenna at the $l^{t h}$ PU over the $n^{t h}$ subcarrier. $\sum_{l=1}^{L} J_{k l}^{n}$ can be modeled as AWGN, which is a general assumption in this research area (e.g. [31] and references therein). This assumption is justified using the central limit theorem. Therefore, $\sigma_{k}^{n 2}=\sigma_{A W G N}^{2}+\sum_{l=1}^{L} J_{k l}^{n}$. Since $\mathbf{U}_{k}^{n \mathrm{H}} \mathbf{H}_{k k}^{n} \mathbf{V}_{k}^{n}$ is considered as the effective channel and has a rank of $d$, the sum rate in (7) can be formulated using spectral decomposition into

$$
R_{k}^{n}\left(\mathbf{H}_{k k}^{n}, P_{k, n}(i)\right)=\sum_{i=1}^{d} \log \left(1+\frac{P_{k, n}(i) \lambda_{i}^{2}\left(\mathbf{U}_{k}^{n \mathrm{H}} \mathbf{H}_{k k}^{n} \mathbf{V}_{k}^{n}\right)}{\sigma_{k}^{n 2}}\right),
$$

where $\lambda_{i}\left(\mathbf{U}_{k}^{n \mathrm{H}} \mathbf{H}_{k k}^{n} \mathbf{V}_{k}^{n}\right)$ is the $i^{\text {th }}$ eigenvalue of $\mathbf{U}_{k}^{n \mathrm{H}} \mathbf{H}_{k k}^{n} \mathbf{V}_{k}^{n}$. Further, we denote $\lambda_{i}\left(\mathbf{U}_{k}^{n \mathrm{H}} \mathbf{H}_{k k}^{n} \mathbf{V}_{k}^{n}\right)$ as $\lambda_{k, i}^{n}$.

The interference introduced by the $k^{\text {th }}$ SU transmitter over the $n^{\text {th }} \mathrm{CR}$ subcarrier transmission to the $l^{\text {th }}$ PU receiver can be expressed as [32]

$$
I_{l k}^{n}\left(D n, P_{k, n}\right)=\sum_{m=1}^{M_{T}} \sum_{i=1}^{M_{P}}\left(\int_{D n-W_{l} / 2}^{D n+W_{l} / 2}\left|g_{l k}^{n, m, i}\right|^{2} P_{k, n, m} \Phi^{n}(f) d f\right),
$$


where $g_{l k}^{n, i, m}$ denotes the channel gain between the the $m^{t h}$ antenna of the $k^{t h}$ SU transmitter and the $i^{t h}$ antenna at the $l^{\text {th }}$ PU receiver over the $n^{\text {th }}$ subcarrier, $P_{k, n, m}$ denotes the power transmitted from the $m^{\text {th }}$ transmit antenna of the $k^{t h}$ SU over the $n^{\text {th }}$ subcarrier, and $\Phi^{n}$ is the PSD of the $n^{\text {th }}$ subcarrier. (10) can be reformulated into [16]

$$
I_{l k}^{n}\left(D n, P_{k, n}\right)=\operatorname{Tr}\left(\Omega_{l}^{n} \mathbf{G}_{l k}^{n} \mathbf{V}_{k}^{n} \mathbf{S}_{k}^{n} \mathbf{V}_{k}^{n \mathrm{H}} \mathbf{G}_{l k}^{n \mathrm{H}}\right),
$$

where $\mathbf{G}_{l k}^{n} \in \mathbb{C}^{M_{P} \times M_{T}}$ denotes the channel gain between the the $k^{\text {th }}$ SU transmitter and the $l^{\text {th }}$ PU over the $n^{t h}$ subcarrier, and $\Omega_{l}^{n}$ is the interference factor of the $l^{t h}$ subcarrier to the $l^{t h}$ PU, which is represented as

$$
\Omega_{l}^{n}=\int_{D n-W_{l} / 2}^{D n+W_{l} / 2} \Phi^{n}(f) d f .
$$

It is also assumed that all the CR system has the perfect information of interference channel gains $\mathbf{G}_{l k}^{n}$. Practically, the CR system is able to obtain the information through periodic sensing of pilot signal from the primary system by assuming the channel reciprocity [33], [34].

The objective of the resource management in this work is to maximize the total throughput of the CR system subject to the interference introduced to the PUs and transmit power budget constraints considering also per-SU minimum rate constraints. IA allows SUs to share the spectrum resources simultaneously, which increases the DoF of the CR system. However, this advantage of using IA is restricted by the IA feasibility condition in (4) since perfect IA can be attained up to a certain number of SUs $\bar{K}$, where $\bar{K}=\frac{M_{T}+M_{R}}{d}-1$. Therefore, the formulation of IA based resource allocation problem should consider this limitation by scheduling only $\bar{K}$ SUs, in which IA is feasible, to share a given subcarrier. Furthermore, the interference from SUs to PUs should be considered in the formulation since no coordination is assumed between the CR and the primary system. The problem can be formulated as

$$
\begin{aligned}
P 1 & : \max _{\mathbf{S}_{k}^{n}, w_{k}^{n}} \sum_{n=1}^{N} \sum_{k=1}^{K} w_{k}^{n} R_{k}^{n}\left(\mathbf{H}_{k k}^{n}, \mathbf{S}_{k}^{n}\right) \\
\text { S.t. : } & \sum_{n=1}^{N} w_{k}^{n} \operatorname{Tr}\left(\mathbf{S}_{k}^{n}\right) \leq P_{k} \quad \forall k \\
& \mathbf{S}_{k}^{n} \succeq 0, \quad \forall n \text { and } \forall k \\
& \sum_{n=1}^{N} \sum_{k=1}^{K} w_{k}^{n} \Omega_{l}^{n} \operatorname{Tr}\left(\mathbf{G}_{l k}^{n} \mathbf{V}_{k}^{n} \mathbf{S}_{k}^{n} \mathbf{V}_{k}^{n \mathrm{H}} \mathbf{G}_{l k}^{n \mathrm{H}}\right) \leq I_{t h}^{l}, \quad \forall l \\
& w_{k}^{n} \in\{0,1\} \forall k, n \\
& \sum_{k=1}^{K} w_{k}^{n}=\bar{K} \forall n \\
& \sum_{n=1}^{N} w_{k}^{n} R_{k}^{n}\left(\mathbf{H}_{k k}^{n}, \mathbf{S}_{k}^{n}\right) \geq R_{\min }, \forall k,
\end{aligned}
$$

where $w_{k}^{n}$ is a binary variable that indicates whether the $n^{\text {th }}$ subcarrier is allocated to the $k^{t h}$ SU or not. $w_{k}^{n}=1$ if and only if the $n^{t h}$ subcarrier is allocated to the $k^{t h} \mathrm{SU}$ and zero otherwise. The constraint (13b) represents the $k^{\text {th }} \mathrm{SU}$ total power constraint $\left(P_{k}\right)$, while a positive transmission power at data stream is guaranteed by (13c). The constraint (13d) ensures that the total interference induced by SUs to the $l^{\text {th }} \mathrm{PU}$ is below the prescribed interference threshold $I_{t h}^{l}$. The equality condition $\sum_{k=1}^{K} w_{k}^{n}=\bar{K}$ ensures that any given subcarrier can be shared by $\bar{K}$ SU links, where IA feasibility is accomplished and, consequently, perfect IA can be achieved. The constraint in (13g) ensures that the fairness among SUs is guaranteed by 
assuming that every $\mathrm{SU}$ has a minimum instantaneous rate $R_{\min }$.

The optimization problem in $P 1$ is a mixed-integer optimization problem, where the mixed-integer nature comes from the integer constraint in (13e) that is used for SUs scheduling. Moreover, the minimum throughput constraints in (13g) increase the complexity of the problem since the CR system may not be able to satisfy this minimum rate due to the limitation introduced by the interference and power budget constraints as well as the channel qualities. Therefore, the complexity of the optimal scheme is generally prohibitive as detailed in Section V. To solve the resource allocation Problem $P 1$ efficiently with low computational complexity, a two-phase sub-optimal algorithm is proposed. In the first phase, for overloaded secondary systems where the number of SUs doesn't satisfy IA feasibility conditions, IA frequency-clustering is performed in order to schedule $\bar{K}$ SUs per subcarrier with fairness consideration. This phase can guarantee feasible and perfect IA on each subcarrier [35], [36]. Afterwards, the available power is distributed among users and subcarriers without violating the interference constraints in the second phase. Moreover, the minimum throughput constraints in (13g) are relaxed by minimizing the number SUs whose rates are below the minimum, i.e. reducing the outage probability of having SUs whose rates are below the minimum. In the sequel, detailed description of the two phases is provided.

\section{Phase I: Frequency-Clustering With Fairness Consideration}

This phase needs to be performed in case of having an overloaded CR system, i.e. the number of SUs, $K$, doesn't satisfy the IA feasibility conditions. As the perfect IA can not be obtained in this case, frequency-clustering algorithm can be executed to cluster the SUs into feasible groups from IA point of view. As an example for frequency-clustering, consider that 6 SUs are operated with $M_{R}=M_{T}=2$ and $d=1$ over $N=4$ subcarriers as seen in Fig.2. This network is considered overloaded since IA is only feasible for 3 SUs. Therefore, IA frequency-clustering is performed and the users are scheduled according to the numbers above the arrows in Fig.2. This means that $\mathrm{SU}_{1}, \mathrm{SU}_{2}$, and $\mathrm{SU}_{4}$ are scheduled to use the first subcarrier while the second subcarrier is shared between $\mathrm{SU}_{2}, \mathrm{SU}_{3}$, and $\mathrm{SU}_{6}$.

In this section, we propose an algorithm for frequency-clustering operation by considering not only their channel quality and per-user power budget constraints but considering also the induced interference to the PU band. This can achieve better sum rate performance for the CR system in interference-limited and power-limited regimes. Moreover, fairness among SUs is guaranteed by assuming that every SU has a minimum instantaneous rate $R_{\min }$.

In order to consider the power-limited regime as well as the interference-limited regime, two initial power distributions are assumed. These power distributions only benefit the clustering operation, where the actual power allocation is executed in the second phase. In the power-limited regime, the power allocation among the subcarriers is mainly restricted by the SUs power budgets, where the interference threshold is relaxed. In this case and assuming that all the SUs are allocated to equal number of subcarriers, the power budget of each SU is equally distributed among the subcarriers, where the allocated power for the $k^{t h}$ user at the $n^{t h}$ subcarrier is expressed as

$$
P_{k, n}^{\mathrm{UF}}=\frac{K P_{k}}{\bar{K} N}
$$

In the interference-limited regime, the power allocation is mainly restricted by the interference threshold of the primary system, where the SUs cannot utilize their power budget since using the full-power budget will induce more interference to the primary system. Hence, we assume that the generated interference to the primary system, i.e. $I_{t h}^{l}$, is equally distributed among the different subcarriers [31]. Consequently, by using (11) and (12), the maximum power, $P_{k, n}^{\mathrm{D}}$, that can be allocated to the $n^{t h}$ subcarrier at the $k^{t h}$ $\mathrm{SU}$ is

$$
P_{k, n}^{\mathrm{D}}=\frac{d I_{t h}^{l}}{N \bar{K} \Omega_{l}^{n} \operatorname{Tr}\left(\mathbf{V}_{k}^{n \mathrm{H}} \mathbf{G}_{l k}^{n \mathrm{H}} \mathbf{G}_{l k}^{n} \mathbf{V}_{k}^{n}\right)} .
$$

The description of the clustering phase can be commenced by defining $\mathcal{A}$ and $\mathcal{N}$ to be the sets that contain all the non-assigned subcarriers and assigned subcarriers, respectively. $\mathcal{B}$ is the set that contains all 


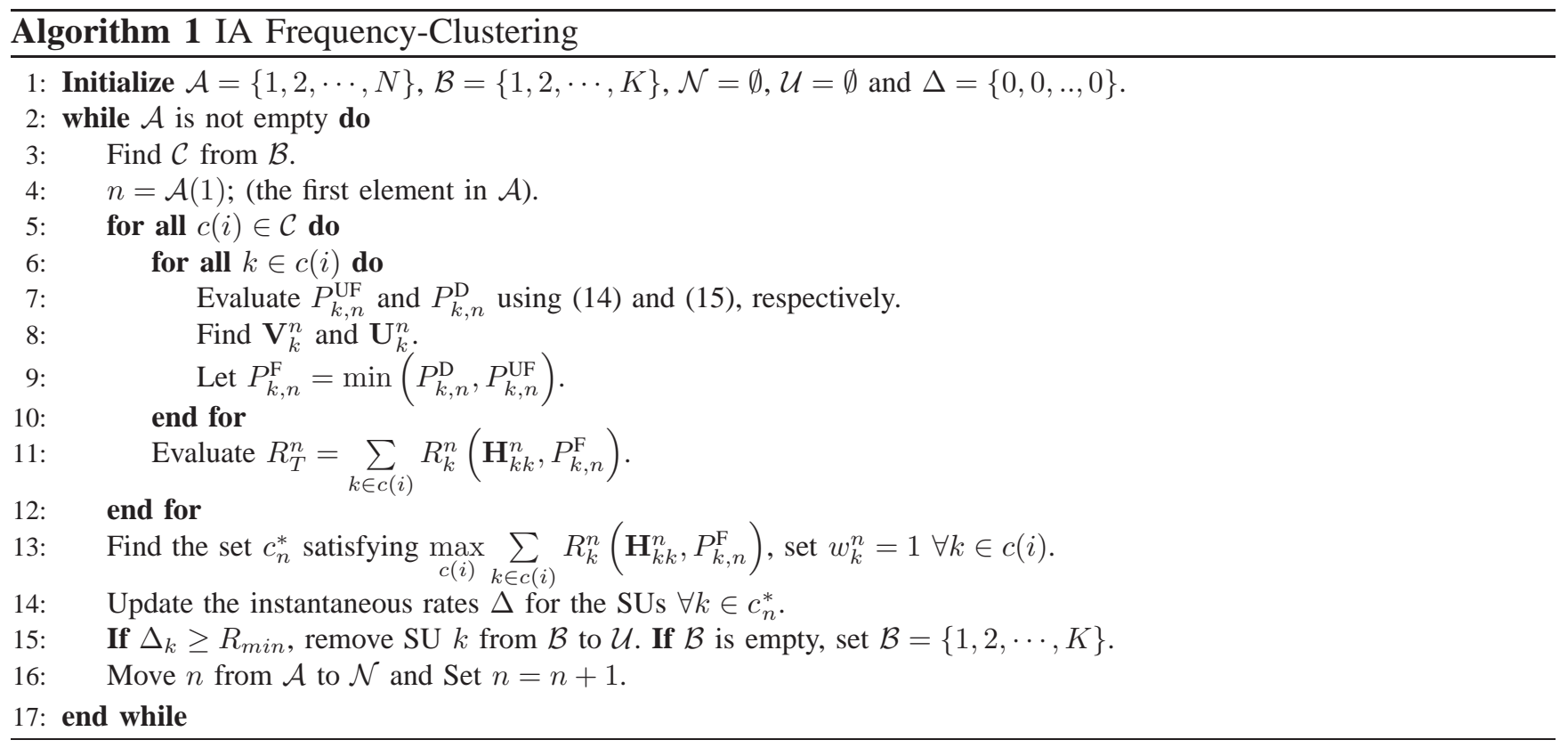

SUs whose rates are below $R_{\text {min }}$, and $\mathcal{U}$ is the set of SUs whose rates are greater than $R_{\text {min }}$. Furthermore, define $\mathcal{C}=\left\{c(1), . ., c\left(A_{\mathcal{C}}\right)\right\}$ to be the sets of all possible clustering combinations where $A_{\mathcal{C}}$ refers to the number of clusters while $c(i) \in \mathcal{C}$ refers to the group of SUs inside the $i^{\text {th }}$ cluster. Each cluster has $\bar{K}$ SUs and hence, $\mathcal{C}$ can be formed by generating all the possible combinations of $\bar{K}$ SUs from the SUs in the set $\mathcal{B}$. Each cluster must satisfy that $c(i) \neq c(j) ; \forall(i \neq j)$. Moreover, we define $\Delta=\left\{\Delta_{1}, . ., \Delta_{K}\right\}$ to be the instantaneous rates for all SUs.

For each subcarrier, the cluster that has the maximum sum rate after performing IA is selected considering the power-limited and interference-limited regimes. For a specific subcarrier, we determine to which regime a given $\mathrm{SU}$ is restricted. If $P_{k, n}^{\mathrm{D}}$ exceeds $P_{k, n}^{\mathrm{UF}}$, i.e. $P_{k, n}^{\mathrm{D}} \geq P_{k, n}^{\mathrm{UF}}$, then the power allocation for the $\mathrm{SU}$ is power-limited and, hence, the allocated power, $P_{k, n}^{\mathrm{F}}$, is fixed to $P_{k, n}^{\mathrm{UF}}$. Otherwise, the power allocation is interference-limited and the allocated power, $P_{k, n}^{\mathrm{F}}$, is fixed to $P_{k, n}^{\mathrm{D}}$. Hence, the considered allocated power in clustering operation can be expressed as

$$
P_{k, n}^{\mathrm{F}}=\min \left(P_{k, n}^{\mathrm{D}}, P_{k, n}^{\mathrm{UF}}\right) .
$$

Accordingly, for the $n^{\text {th }}$ subcarrier, the cluster selection process can be formulated mathematically to select $c_{n}^{*}$ as

$$
c_{n}^{*}=\max _{c(i)} \sum_{k \in c(i)} R_{k}^{n}\left(\mathbf{H}_{k k}^{n}, P_{k, n}^{\mathrm{F}}\right) .
$$

The users inside this cluster are the only allowed SUs in the system to transmit over that subcarrier.

To describe the selection mechanism, the algorithm starts by allocating the subcarriers that are located next to the PU band moving towards the distant ones since the subcarriers close to the PU bands will potentially use low transmit power even that they have good channel conditions. Keeping those subcarriers to the end of the assignment in the frequency clustering algorithm will make them suffer not only from the transmission power limitation but also from the low diversity in choosing the users from the set of users whose instantaneous rate below the minimum. The subcarriers are assigned sequentially to clusters. Initially, the possible cluster combinations are generated using the SUs in the set $\mathcal{B}$, where $\mathcal{B}$ is assumed to contain all SUs at the beginning. Throughout the allocation of the different subcarriers, if the rate of the $k^{\text {th }}$ SU becomes more than the minimum required rate $R_{\min }$, the user will be removed form the set $\mathcal{B}$ to the set $\mathcal{U}$. If the minimum rate constraints are satisfied for all the users, i.e. $\mathcal{B}$ is empty, the subcarrier can be allocated to one of the clusters that are generated from SUs in the set $\mathcal{U}$, which will contain all 
SUs at this moment. If the optimization problem is assumed to be solved without any minimum rate constraints, the set $\mathcal{U}$ will be assumed always empty and accordingly the subcarrier can be allocated to any one of the SUs. To allocate a given subcarrier, the algorithm initially forms all cluster combinations of the SUs in the set $\mathcal{B}$ and evaluates the allocated power, $P_{k, n}^{\mathrm{F}}$. Afterwards, the subcarrier is allocated to the cluster $c_{n}^{*}$ that achieves the maximum sum rate according to (17) and, then, removed to $\mathcal{N}$. Afterwards, the instantaneous rates, $\Delta$, of the SUs in $c_{n}^{*}$ is updated and the SUs whose rates are greater that the minimum required rate, $R_{\text {min }}$, are removed form the set $\mathcal{B}$ to the set $\mathcal{U}$. The scheme is repeated until the allocation of all subcarriers in order to find the selected clusters for all subcarriers, $\mathcal{X}=\left\{c_{1}^{*}, . ., c_{N}^{*}\right\}$. The clustering procedures are summarized in Algorithm 1.

\section{Phase II:Power Allocation Algorithm}

By performing the frequency-clustering phase, the subcarriers are allocated to the different clusters. Therefore, the subcarrier indicators $w_{k}^{n}$ are already determined from the previous phase. Therefore, the power allocation problem can be formulated as follows

$$
\begin{aligned}
P 2: & \max _{P_{k, n}(i)} \sum_{n=1}^{N} \sum_{k \in c_{n}^{*}} \sum_{i=1}^{d} \log \left(1+\frac{P_{k, n}(i) \lambda_{k, i}^{n}}{\sigma_{k}^{n 2}}\right) \\
\text { s.t. : } & \sum_{n=1}^{N} \sum_{i=1}^{d} P_{k, n}(i) \leq P_{k} \quad \forall k \\
& P_{k, n}(i) \geq 0, \quad \forall n \text { and } \forall k \\
& \sum_{n=1}^{N} \sum_{k \in c_{n}^{*}} \sum_{i=1}^{d} \Omega_{l}^{n} P_{k, n}(i) \bar{g}_{k}^{n}(i) \leq I_{t h}^{l}, \quad \forall l,
\end{aligned}
$$

where $\bar{g}_{k}^{n}(i)$ is the $i^{\text {th }}$ element in the diagonal of matrix $\overline{\mathbf{G}}_{k}^{n}=\mathbf{V}_{k}^{n \mathrm{H}} \mathbf{G}_{l k}^{n \mathrm{H}} \mathbf{G}_{l k}^{n} \mathbf{V}_{k}^{n}$. It is worth to highlight that for the $n^{\text {th }}$ subcarrier the SUs inside the cluster $c_{n}^{*}$ are the only considered in the power allocation phase.

The problem $P 2$ is a convex optimization problem. The Lagrangian can be written as

$$
\begin{aligned}
\mathcal{L} & =-\sum_{n=1}^{N} \sum_{k \in c_{n}^{*}} \sum_{i=1}^{d} \log \left(1+\frac{1}{\sigma_{k}^{n 2}} P_{k, n}(i) \lambda_{k, i}^{n}\right)+\sum_{k=1}^{K} \beta_{k}\left(\sum_{n=1}^{N} \sum_{i=1}^{d} P_{k, n}(i)-P_{k}\right) \\
& +\sum_{l=1}^{L} \alpha^{l}\left(\sum_{n=1}^{N} \sum_{k \in c_{n}^{*}} \sum_{i=1}^{d} \Omega_{l}^{n} P_{k, n}(i) \bar{g}_{k}^{n}(i)-I_{t h}^{l}\right)-\sum_{n=1}^{N} \sum_{k=1}^{K} \sum_{i=1}^{d} P_{k, n}(i) \mu_{k}^{n},
\end{aligned}
$$

where $\beta_{k}, \alpha^{l}$ and $\mu_{k}^{n}$ are the non-negative Lagrange multipliers. The Karush-Kuhn-Tucker (KKT) conditions can be described as follows

$$
\begin{aligned}
& P_{k}^{n} \geq 0 ; \beta_{k} \geq 0 ; \alpha^{l} \geq 0 ; \mu_{k}^{n} \geq 0 \\
& \beta_{k}\left(\sum_{n=1}^{N} \sum_{i=1}^{d} P_{k, n}(i)-P_{k}\right)=0, \forall k \\
& \alpha^{l}\left(\sum_{n=1}^{N} \sum_{k \in c_{n}^{*}} \sum_{i=1}^{d} \Omega_{l}^{n} P_{k, n}(i) \bar{g}_{k}^{n}(i)-I_{t h}^{l}\right)=0, \forall l \\
& \frac{\partial \mathcal{L}}{\partial P_{k, n}(i)}=\frac{-1}{\frac{\sigma_{k}^{n 2}}{\lambda_{k, i}^{n}}+P_{k, n}(i)}+\sum_{k=1}^{K} \beta_{k}+\sum_{l=1}^{L} \alpha^{l} \Omega_{l}^{n} \bar{g}_{k}^{n}(i)-\mu_{k}^{n}=0 .
\end{aligned}
$$


After rearranging (20d), we get

$$
P_{k, n}(i)=\left[\frac{1}{\sum_{l=1}^{L} \alpha^{l} \Omega_{l}^{n} \bar{g}_{k}^{n}(i)+\sum_{k=1}^{K} \beta_{k}}-\frac{\sigma_{k}^{n 2}}{\lambda_{k, i}^{n}}\right]^{+},
$$

where $[y]^{+}=\max (0, y)$. The optimal solution of problem $P 2$ requires high computational complexity and its complexity grows exponentially with the number of subcarriers. This prompts to propose a low complexity algorithm approaching the optimal solution. Therefore, we propose the sub-optimal power allocation algorithm that distributes the power among the subcarriers and SUs with less complexity.

In the rest of this work, to make the analysis more clear and without loss of generality, we assume that each SU sends one data stream to its intended receiver. Accordingly, $\bar{g}_{k}^{n}=\mathbf{V}_{k}^{n \mathrm{H}} \mathbf{G}_{l k}^{n \mathrm{H}} \mathbf{G}_{l k}^{n} \mathbf{V}_{k}^{n}$. Moreover, the sum rate in (7) can be written as

$$
R_{k}^{n}=\log \left(1+\frac{1}{\sigma_{k}^{n 2}} P_{k, n} \bar{h}_{k}^{n}\right)
$$

where $\bar{h}_{k}^{n} \triangleq \mathbf{U}_{k}^{n \mathrm{H}} \mathbf{H}_{k k}^{n} \mathbf{V}_{k}^{n} \mathbf{V}_{k}^{n \mathrm{H}} \mathbf{H}_{k k}^{n}{ }^{\mathrm{H}} \mathbf{U}_{k}^{n}$.

Further, the sub-optimal power allocation is described through four steps, where this method allocates the power in a novel way by dividing Problem $P 2$ into two sub-problems: power allocation problem considering only interference constraint and, then, a cap-limited waterfilling problem considering only the power budget of SUs. Accordingly, the power can be allocated to SUs and subcarriers as stated in the following stages.

1) Finding the maximum power: the maximum power $P_{k, n}^{\max }$ that can be allocated to the $k^{\text {th }}$ user over the $n^{\text {th }}$ subcarrier is determined by ignoring the per-SU power constraints and considering only the interference constraints. Therefore, by considering only the $l^{\text {th }}$ PU interference constraint, the problem is reduced to

$$
\begin{aligned}
P 3 & : \max _{\widehat{P}_{k, n}} \sum_{n=1}^{N} \sum_{k \in c_{n}^{*}} \log \left(1+\frac{1}{\sigma_{k}^{n 2}} \widehat{P}_{k, n} \bar{h}_{k}^{n}\right) \\
\text { s.t. : } & \sum_{n=1}^{N} \sum_{k \in c_{n}^{*}} \Omega_{l}^{n} \widehat{P}_{k, n} \bar{g}_{k}^{n} \leq I_{t h}^{l} \\
& \widehat{P}_{k, n} \geq 0, \quad \forall n \text { and } \forall k,
\end{aligned}
$$

where $(\widehat{\cdot})$ represents the variables that are optimized under the interference constraint only. By solving $P 3 ; \forall l \in\{1,2, \cdots, L\}$, we obtain

$$
\widehat{P}_{k, n}^{l}=\left[\frac{1}{\widehat{\alpha}^{l} \Omega_{l}^{n} \bar{g}_{k}^{n}}-\frac{\sigma_{k}^{n 2}}{\bar{h}_{k}^{n}}\right]^{+},
$$

where the Lagrange multiplier $\widehat{\alpha}^{l}$ is evaluated using (24) and (23b) as

$$
\widehat{\alpha}^{l}=\frac{|N K|}{I_{t h}^{l}+\sum_{n=1}^{N} \sum_{k \in c_{n}^{*}} \frac{\Omega_{l}^{n} \sigma_{k}^{n 2} \bar{g}_{k}^{n}}{h_{k}^{n}}} .
$$

By solving $P 3$ for every interference constraint, $P_{k, n}^{\max }$ is evaluated as

$$
P_{k, n}^{\max }=\min \left\{\widehat{P}_{k, n}^{l}\right\}_{l=1}^{L} .
$$


By applying this formula, one can guarantee that the interference introduced to the PU bands is below the maximum limit. This step is expressed graphically in Fig. 3.a, where the maximum power that can be allocated for each subcarrier over the SUs is expressed by the red lines.

2) Checking power budget constraints: when the maximum power $P_{k, n}^{\max }$ is determined, the per-SU power constraints are tested. If the relation $\sum_{n=1}^{N} P_{k, n}^{\max } \leqslant P_{k}$ is satisfied for all SUs, the optimal solution of the optimization problem $P 2$ is determined to be $\bar{P}_{k, n}=P_{k, n}^{\max }$ which is equal to the maximum power that can be allocated to each subcarrier. Otherwise, proceed to the next steps.

3) Distribution of the power budgets: the power budget $P_{k}$ for each SU is distributed among its allocated subcarriers subject to be lower that or equal to the power upper-bound of each user at each subcarrier $P_{k, n}^{\max }$. The problem is formulated as a cap-limited waterfilling problem as follows [37]

$$
\begin{aligned}
& P 4: \max _{\widetilde{P}_{k, n}} \sum_{n=1}^{N} \sum_{k \in c_{n}^{*}} \log \left(1+\frac{1}{\sigma_{k}^{n 2}} \widetilde{P}_{k, n} \bar{h}_{k}^{n}\right) \\
& \text { s.t. : } \sum_{n=1}^{N} \widetilde{P}_{k, n} \leq P_{k} \\
& \quad 0 \leq \widetilde{P}_{k, n} \leq P_{k, n}^{\max },
\end{aligned}
$$

where $\widetilde{P}_{k, n}$ is the allocated power by solving problem $P 4$. This problem can be solved efficiently using a successive application of the conventional waterfilling concept. As a starting point, the waterfilling solution is found as [38]

$$
\widetilde{P}_{k, n}^{\mathrm{WF}}=\left[\lambda-\frac{\sigma_{k}^{n 2}}{\bar{h}_{k}^{n}}\right]^{+},
$$

where $\widetilde{P}_{k, n}^{\mathrm{WF}}$ is the allocated power by waterfilling solution for the $k^{\text {th }}$ user at the $n^{\text {th }}$ subcarrier, and $\lambda$ is the waterfilling level. Thereafter, if the power allocated by waterfilling solution $\widetilde{P}_{k, n}^{\mathrm{WF}}$ is greater than $P_{k, n}^{\max }$, the power is readjusted to $P_{k, n}^{\max }$ and the already allocated power is subtracted from the total power budget. Then, successive waterfilling is performed over the users and subcarriers that did not exceed the maximum power $P_{k, n}^{\max }$ in the last step until reaching the iteration in which $\widetilde{P}_{k, n}$ doesn't exceed $P_{k, n}^{\max }$ for any user and subcarrier. This step is described graphically in Fig. 3.b, where the highlighted colours represent the power that is allocated using cap-limited waterfilling.

4) Re-adjustment of the power levels: the allocated power per subcarrier $\widetilde{P}_{k, n}$ found by solving $P 4$ is less than or equal $P_{k, n}^{\max }$. Therefore, some of the allocated power $\widetilde{P}_{k, n}$ doesn't not reach the maximum allowed power. Consequently, the system loses some of the allowed power resources as the interference constraint is not satisfied with equality which decreases the capacity of CR system. Therefore, some power can be moved from one subcarrier to another in order to enhance system throughput. This can be achieved by updating the maximum power that can be allocated to each subcarrier $P_{k, n}^{\max }$ depending on the residual interference $I_{R}^{l}$, which can be calculated as follows

$$
I_{R}^{l}=I_{t h}^{l}-\sum_{n=1}^{N} \sum_{k \in c_{n}^{*}} \widetilde{P}_{k, n} \Omega_{l}^{n} \bar{g}_{k}^{n} .
$$

Assuming that $B_{l}$ is the set of subcarriers that reach the maximum allowed power, i.e. $\widetilde{P}_{k, n}=$ $P_{k, n}^{\max } ; \forall n \in B_{l}$, then, $P_{k, n}^{\max } ; \forall n \in B_{l}$ can be updated by applying the equations (24)-(26) on the subcarriers in the set $B_{l}$ with the updated interference constraints, which can be evaluated as

$$
I_{t h}^{\prime l}=I_{R}^{l}+\sum_{n \in B_{l}} \sum_{k \in B_{l}} \widetilde{P}_{k, n} \Omega_{l}^{n} \bar{g}_{k}^{n} .
$$



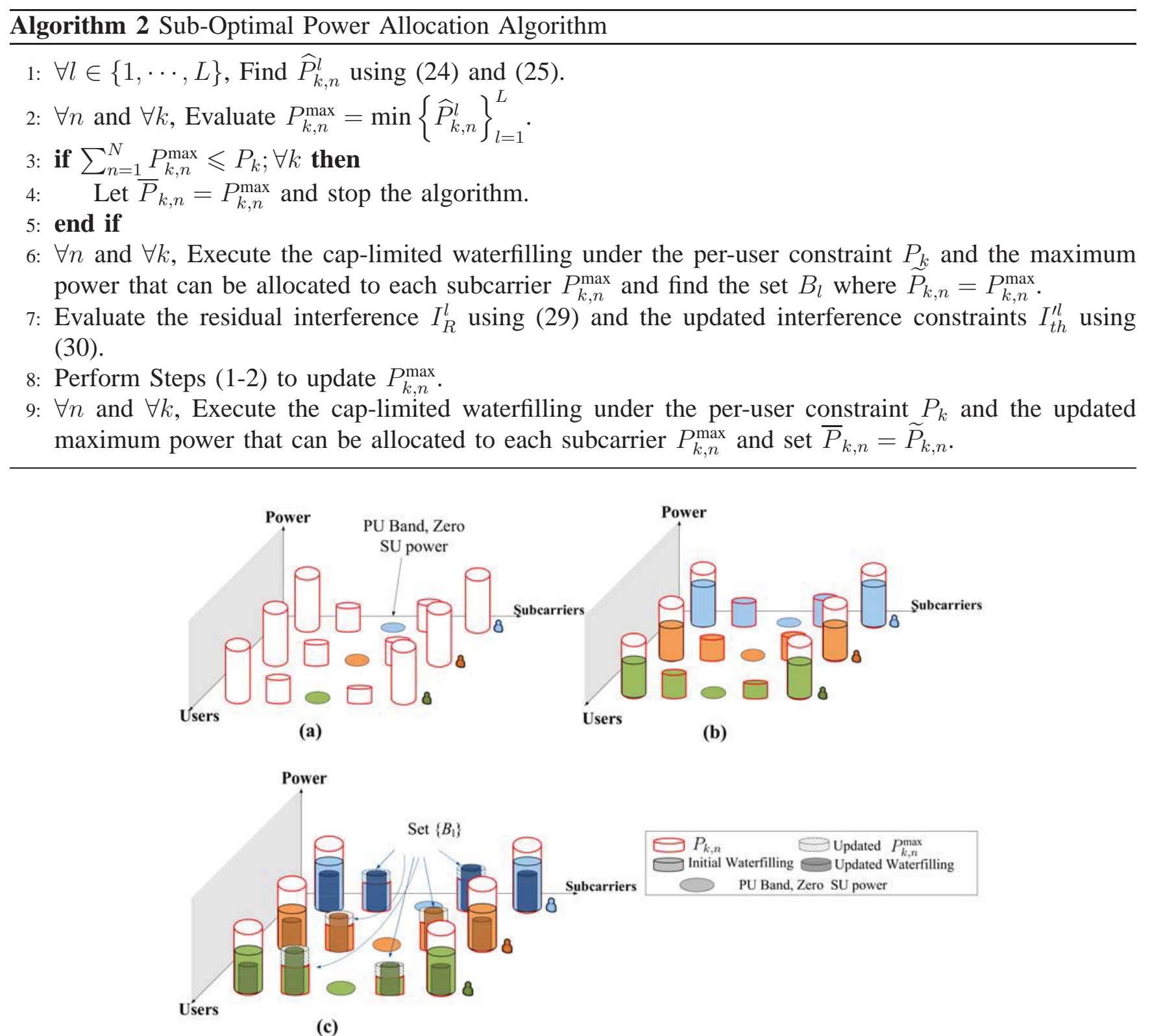

Fig. 3: Graphical representation of the proposed power allocation algorithm.

Finally, the procedures of the cap-limited waterfilling that were used to solve problem $P 4$ is reperformed to find the final solution $\bar{P}_{k, n}=\widetilde{P}_{k, n}$. At this point, the solution $\bar{P}_{k}^{n}$ is approaching the optimal solution and satisfying the interference constraints with equality as well as guaranteeing that the total power budget constraints are satisfied. Fig. 3.c summarizes the procedures of this step graphically using the dashed highlighted colours.

The power allocation phase is described in Algorithm 2.

\section{Computational Complexity Analysis}

In this section, we present the computational analysis of the optimal solution and the proposed algorithm. In terms of complexity, the optimal solution that is formulated in Problem $P 1$ needs to iterate $\left(\begin{array}{l}K \\ \bar{K}\end{array}\right)^{N}$ times to exhaust all the cluster combinations of SUs, where the power allocation of Problem $P 2$ is performed and IA solution is computed for each combination. The complexity of IA solution is dependent on the algorithm 
that is used to find IA solution. As an example, minimum leakage interference (MLI) method requires a complexity of $\bar{K} . T .\left[\mathcal{O}\left(M_{T}^{3}\right)+\mathcal{O}\left(M_{R}^{3}\right)\right]+\bar{K} . T .\left[2(\bar{K}-1)\left(\mathcal{O}\left(M_{R} M_{T}^{2}\right)+\mathcal{O}\left(M_{T} M_{R}^{2}\right)\right)\right]$, where $T$ is the number of iterations in the reciprocity channel [39]. Many research work in the literature tackled the problem of designing low complexity solutions for IA as in [40]-[42] and references therein. The design of such solutions is out of the scope of this paper. Therefore, we denote the complexity of finding IA solution by $\Psi$. Accordingly, the computational complexity of the optimal scheme is

$$
\mathcal{O}\left(\left(\begin{array}{l}
K \\
\bar{K}
\end{array}\right)^{N} \cdot\left(\Psi+(\bar{K} d N)^{3}\right)\right)
$$

where $\bar{K} d N$ is the number of the variables that needed to be optimized using the interior point optimization technique.

Since the complexity of the optimal scheme is very hard to afford, the sub-optimal approach is proposed through two phases as discussed before. In the frequency-clustering algorithm, a maximum of $\left(\begin{array}{l}K \\ \bar{K}\end{array}\right)$ IA solutions are found for every subcarrier. Accordingly, the complexity of frequency-clustering phase is

$$
\mathcal{O}\left(\left(\begin{array}{l}
K \\
\bar{K}
\end{array}\right) \cdot N \cdot \Psi\right) \text {. }
$$

Referring to the sub-optimal power allocation in Algorithm 2, step 1 in Algorithm 2 has a waterfilling like computational complexity of $\mathcal{O}(\bar{K} d N \log (\bar{K} d N))$ [43], [44]. Step 1 should be performed for $L$ interference constraints, hence the complexity of step 1 is $\mathcal{O}(L \bar{K} d N \log (\bar{K} d N)) \leq \mathcal{O}(K L \bar{K} d N \log (\bar{K} d N))$. Steps 6 and 9 in the algorithm execute the cap-limited waterfilling for all SUs with a complexity of $\mathcal{O}(\bar{K} d N \log (\bar{K} d N))$. Accordingly, the complexity of steps 6 and 9 is $\mathcal{O}(K \bar{K} d N \log (\bar{K} d N)) \leq$ $\mathcal{O}(K L \bar{K} d N \log (\bar{K} d N))$. Step 8 has a complexity of $\mathcal{O}\left(\left|B_{l}\right| \log \left|B_{l}\right|\right) \leq \mathcal{O}(K L \bar{K} d N \log (\bar{K} d N))$ considering all SUs. Therefore, the computational complexity of the sub-optimal power allocation algorithm is lower than $\mathcal{O}(K L \bar{K} d N \log (\bar{K} d N))$.

Correspondently, the complexity of the proposed sub-optimal resource allocation algorithm through the two phases is lower than $\mathcal{O}\left(\left(\frac{K}{\bar{K}}\right) \cdot N \cdot \Psi+K L \bar{K} d N \log (\bar{K} d N)\right)$, which is much lower than the computational complexity of the optimal solution.

\section{Simulation SEtup and Results}

In our simulation, we investigate the performance of using IA based resource allocation algorithm. The performance is compared with frequency division multiple access (FDMA) technique as an orthogonal transmission scheme in MIMO-OFDM CR network. The CR system is assumed to have $K=12$ SUs with $N=128$ subcarriers. The PSD of the $n^{\text {th }}$ subcarrier of the OFDM system is expressed as [6]

$$
\Phi_{n}(f)=T_{s}\left(\frac{\sin \pi f T_{s}}{\pi f T_{s}}\right)^{2},
$$

where $T_{s}$ is the symbol duration. $M_{T}=M_{R}=2$ antennas at each SU node are assumed while a single antenna at each PU node is considered. Two active PU bands are assumed where each has 10 $\mathrm{MHz}$ bandwidth. Moreover, the non active band is located between the active bands and has $10 \mathrm{MHz}$ of bandwidth. Channel realizations have been drawn from independent and identically distributed Gaussian distribution with zero mean and unit variance. The value of noise variance $\sigma_{k}^{n 2}$ is assumed to be $10^{-6}$. In this scenario, IA solution is evaluated using closed-form solution as in [10], where IA in the considered system is only feasible for $\bar{K}=3 \mathrm{SUs}$. The minimum rate for each SU is set to be $R_{\min }=150$ bits per OFDM symbol.

Obtaining the optimal solution of problem $P 1$ is very hard even for small number of subcarriers and users. For the purpose of performance comparison, the following algorithms are considered in the simulation: 


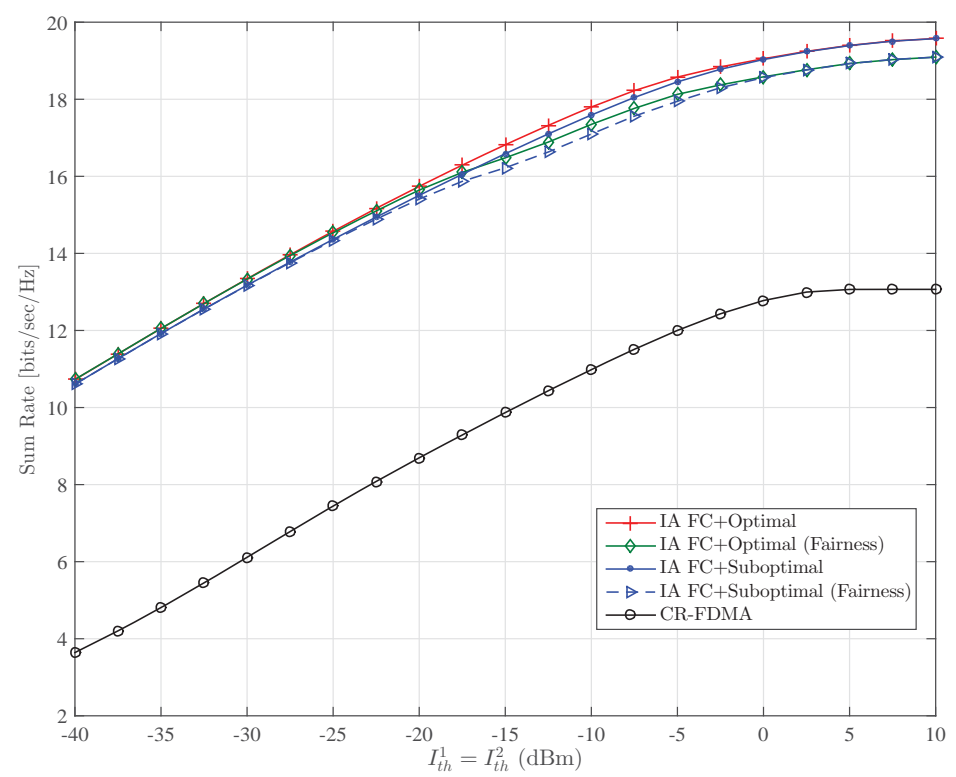

Fig. 4: Achieved sum rate vs. allowed interference threshold.

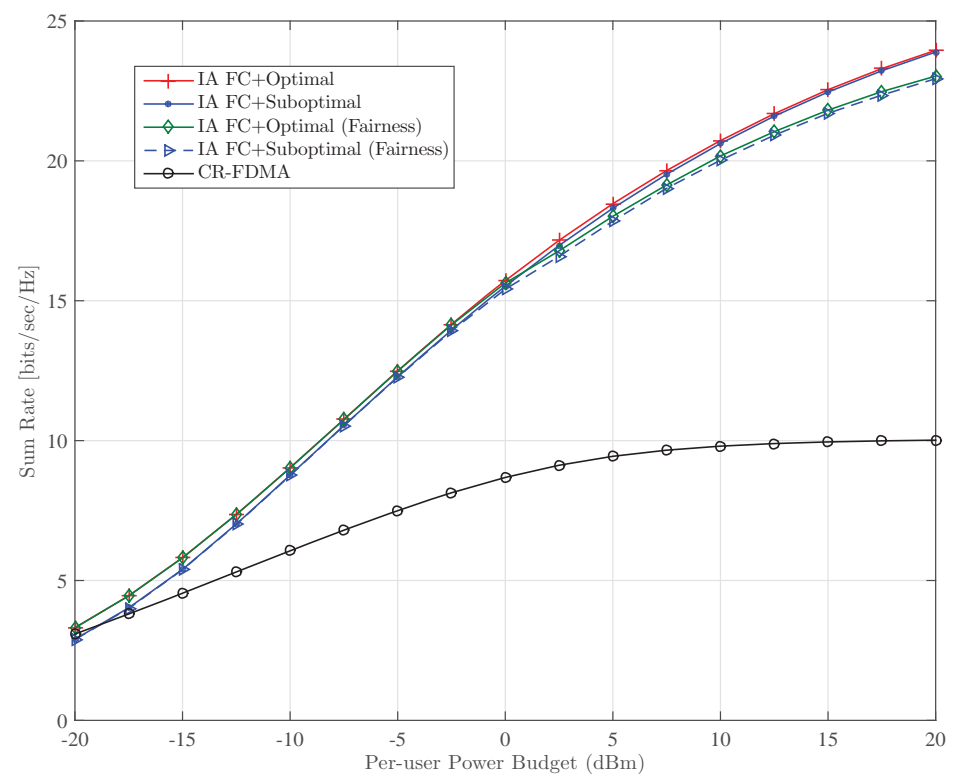

Fig. 5: Achieved sum rate vs. per-SU power budget.

1) IA FC+Optimal: performs frequency-clustering using Algorithm 1 and evaluates the optimal power distribution using CVX toolbox [45]. The word Fairness is added between parenthesis when the fairness constraint is considered.

2) IA FC+Suboptimal: applies frequency-clustering using Algorithm 1 and performs the power allocation based on Algorithm 2. The word Fairness is added between parenthesis when the fairness constraint is considered.

3) CR-FDMA: distributes the different radio resources optimally considering an FDMA system as in [5].

We first show the impact of the interference threshold with $I_{t h}^{1}=I_{t h}^{2}$ on the average sum rate when the per-SU power budget is set to be $P_{k}=0 \mathrm{dBm}$, as shown in Fig. 4 . In general, for all resource allocation methods, the average sum rate increases as the interference threshold levels increase since each SU has 


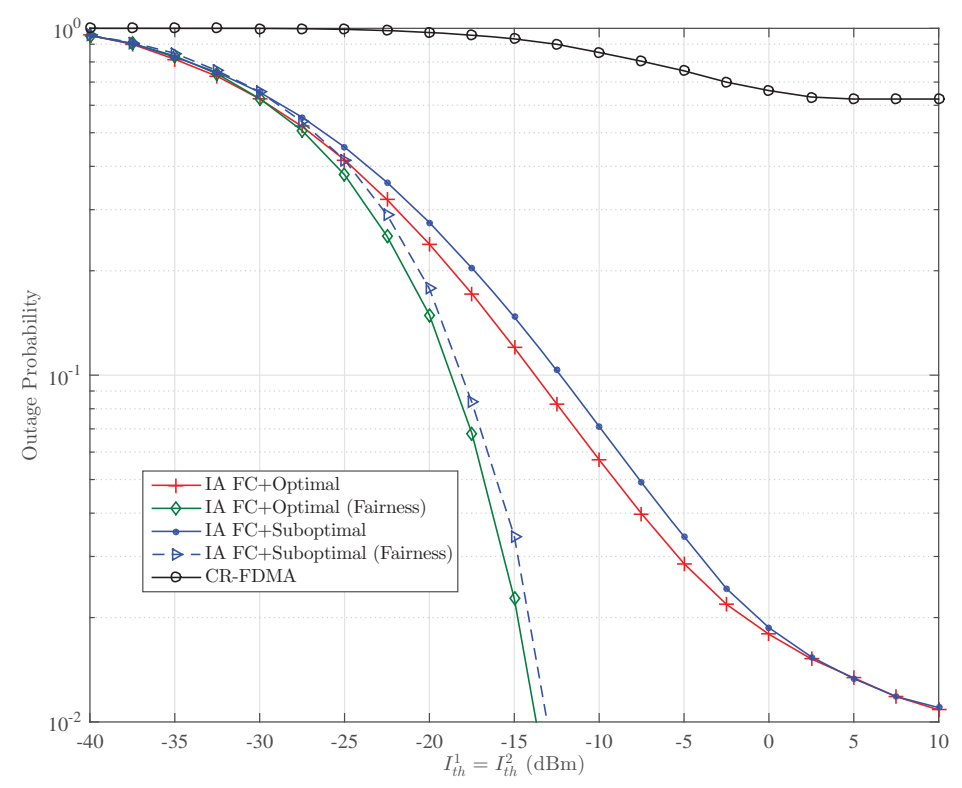

Fig. 6: Outage probability vs. interference thresholds, $R_{\min }=150$ bits/symbol.

more flexibility to allocate more power on its subcarriers. It can be observed also that IA FC+Suboptimal algorithm strictly matches the corresponding curves of IA FC+Optimal, which reveals the efficiency of the sub-optimal algorithm. It can be observed that IA FC+Optimal and IA FC+Suboptimal algorithms achieves higher sum rate in compared with CR-FDMA algorithm. Furthermore, the sum rate increases with the increase of interference threshold until a certain interference threshold value. After this value, the sum rate remains constant as the CR behaves like a non-CR system where the interference constraint has no effect on the optimization problem. In low interference threshold values, the algorithms with fairness perform very close to those without fairness consideration as the fairness constraint can not be achieved with this low interference threshold value. Accordingly, the algorithm acts as there is no fairness constraint. After a certain interference constraint value $(-20 \mathrm{dBm}$ in the figure), the fairness constraint can be satisfied for the users. The loss in the sum rate is because of the activation of the fairness constraint.

The average sum rate versus per-SU power constraint is presented in Fig. 5 where $I_{t h}^{1}=I_{t h}^{2}=-20 \mathrm{dBm}$. The sum rate of the CR systems increases as the per-SU power budget increases up to certain power value, afterwards the sum rate remains constant because the CR system reaches to the maximum power that can be allocated under the interference threshold. The gap between IA based resource allocation algorithms and CR-FDMA increases with the increase of the power constraints, which shows the efficiency of IA in utilizing the available resources. The behavior of the algorithm with fairness constraint consideration has the same interpretation as commented on Fig. 4.

Fig. 6 presents the outage probability of the different algorithms against interference threshold when the per-SU power budget is set to be $P_{k}=0 \mathrm{dBm}$. Generally, the outage probability decreases with the increase of interference constraint as the algorithms become more able to support the instantaneous rate for the different users. Furthermore, the outage probability of the IA FC+Suboptimal is very close to IA $F C+$ Optimal, and both are much lower than that of the CR-FDMA. It is clearly observed from this figure that IA based resource allocation algorithms is able to achieve a high-level of fairness among the different users. The best outage probability is achieved, as expected, by the algorithms when the fairness constraint is considered.

Fig. 7 plots the instantaneous rate for a given user over time when $I_{t h}^{1}=I_{t h}^{2}=-10 \mathrm{dBm}, P_{k}=0 \mathrm{dBm}$ and $R_{\min }=150$ bits per OFDM symbol. It is noted from the figure that the instantaneous rate fluctuates along the time. The high values mean that this user is assigned a larger number of subcarriers compared to others while low values mean that other users have a large number of subcarriers causing the deep rate. 


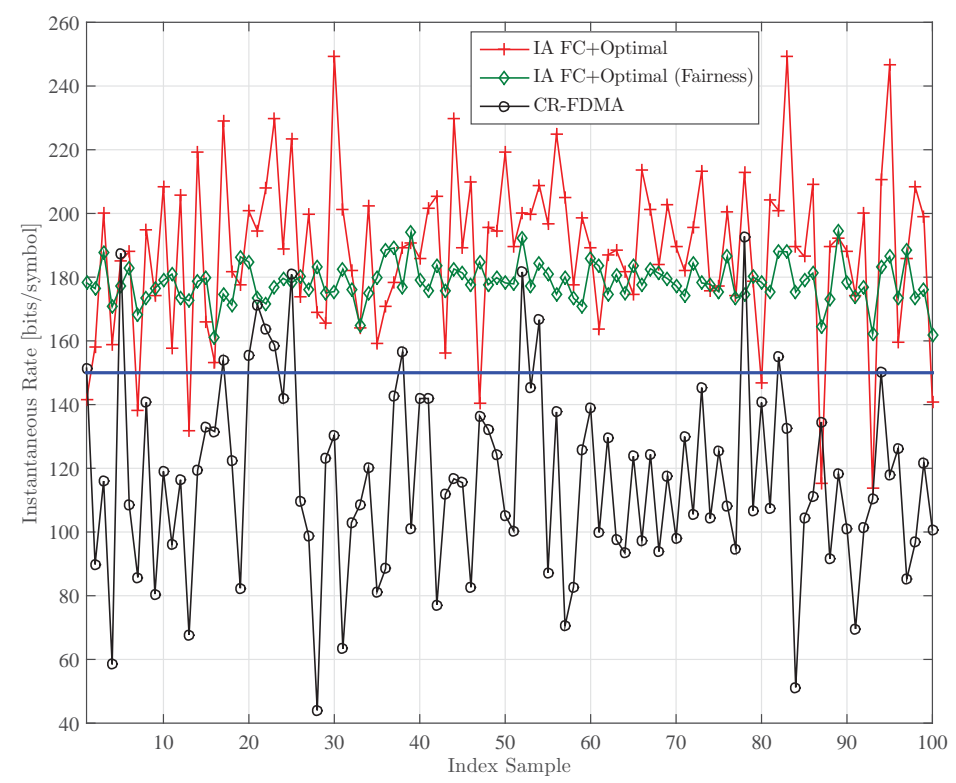

Fig. 7: Achieved Instantaneous Rate.

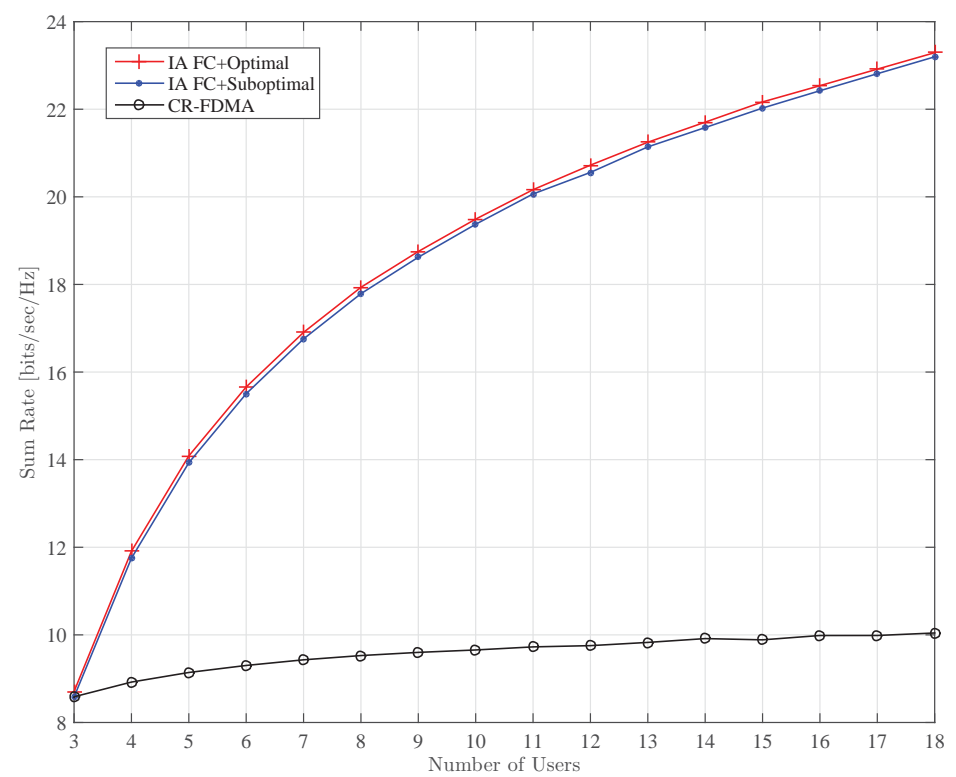

Fig. 8: Achieved sum rate vs. number of SUs.

Therefore, IA based resource allocation exhibits better instantaneous rate compared to CR-FDMA since the fluctuations of $C R-F D M A$ is stronger and changes dramatically, which causes deep rate degradation at some time samples. Moreover, IA FC+Optimal with fairness consideration presents smooth instantaneous rate compared to others, which means that the users get fair allocation of the subcarriers. Clearly, $I A$ FC+Optimal with fairness always achieves a better rate than the minimum unlike the other compared methods.

Fig. 8 presents the average sum rate versus the number of SUs when the interference threshold is $I_{t h}^{1}=I_{t h}^{2}=-20 \mathrm{dBm}$ and the per-SU power budget is $P_{k}=10 \mathrm{dBm}$. Generally, the sum rate increases with the number of SUs due to the increase in the multiuser diversity and the average SNR per subcarrier. Moreover, IA based resource allocation algorithms exploit much more gain from the increase in the multiuser diversity than CR-FDMA as the IA based resource allocation algorithms allows more users to 


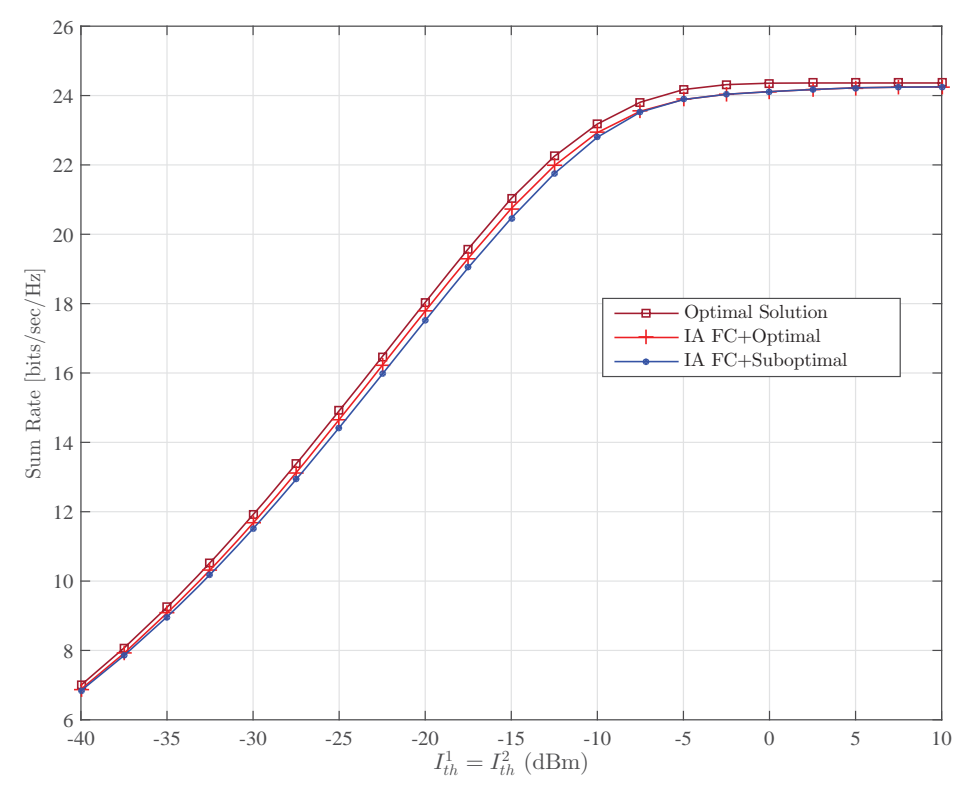

Fig. 9: Achieved sum rate vs. allowed interference threshold when $K=4, P_{k}=0 \mathrm{dBm}$ and $N=4$.

share the available resources.

Finally, we consider a small-scale system of $K=4$ SUs and $N=4$ subcarriers in order to present a comparison between the proposed algorithms and the optimal solution since the complexity of the optimal solution is very high to be performed for the previously simulated scenario. For this small system, 256 different combinations of SUs clusters should be considered for power allocation in the optimal solution. If the number of subcarriers is increased by one (i.e. $N=5$ ), 1024 cluster combinations are required. Additionally, 10000 combinations are required when the number of SUs is increased by one (i.e. $K=5$ ). This clearly present the prohibitive complexity of the optimal solution. Therefore, we use a small scale system of $K=4$ SUs and $N=4$ subcarriers in the comparison. Fig. 9 presents the average sum rate for the optimal solution and the proposed algorithms against the interference thresholds when the perSU power budget is set to be $P_{k}=0 \mathrm{dBm}$. The fairness constraint is not considered since the fairness constraint is not feasible to be achieved through the optimal solution. Fig. 9 presents that the performance of proposed algorithms closely approaches the optimal one with less complexity, which reveals the efficient performance of the proposed algorithm. For the considered small-scale CR system with $K=4$ SUs and $N=4$ subcarriers, the optimal solution requires 256 cluster combinations of SUs in order to find the optimal solution while the IA FC+Optimal needs only 16 cluster combinations to achieve almost the same performance.

\section{CONCLUSION}

In this paper, efficient resource allocation in overloaded MIMO CR systems is achieved using IA with frequency-clustering without affecting the quality of service of the primary system. The problem is formulated as a mixed-integer problem. Furthermore, an efficient sub-optimal algorithm is proposed to reduce the computational complexity of the problem through two phases. In the first phase, frequencyclustering is performed to assign one group of SUs to each subcarrier in order to make IA feasible considering the fairness constraints. While in the second phase, the power is distributed among subcarriers considering the induced interference limits. Simulations show that IA technique achieves a significant sum rate increase of CR systems compared to traditional CR systems (CR-FDMA). With a significant reduction of the computational complexity, the proposed sub-optimal scheme achieves a very close performance to the optimal one. Moreover, the algorithm with fairness consideration presents better fairness among the SUs. 


\section{REFERENCES}

[1] M. El-Absi, M. Shaat, F. Bader, and T. Kaiser, "Interference alignment with frequency-clustering for efficient resource allocation in cognitive radio networks,” in Proc. IEEE Globecom'14, 8-12 Dec. 2014.

[2] I. Akyildiz, L. Won-Yeol, M. C. Vuran, and S. Mohanty, "NeXt generation/dynamic spectrum access/cognitive radio wireless networks: A survey,” Computer Networks, vol. 50, no. 13, pp. 2127 - 2159, 2006.

[3] Z. Hu, G. Zhu, Y. Xia, and G. Liu, "Multiuser subcarrier and bit allocation for MIMO-OFDM systems with perfect and partial channel information,” in Proc. IEEE WCNC’2004, vol. 2, March 2004, pp. 1188-1193.

[4] H. Yongqing, Q. Peng, and H. Shao, "Adaptive bit and power loading algorithm with low complexity in MIMO-OFDM systems," J. Sys. Engineering and Electronics, vol. 19, no. 3, pp. 461-466, June 2008.

[5] R. Zhang and Y. Liang, "Exploiting multi-antennas for opportunistic spectrum sharing in cognitive radio networks,” IEEE J. Sel. Topics Signal Proc., vol. 2, no. 1, pp. 88-102, 2008.

[6] T. Weiss, J. Hillenbrand, A. Krohn, and F. K. Jondral, "Mutual interference in OFDM-based spectrum pooling systems," in Proc. IEEE VTC’04F, vol. 4, 2004, pp. 1873-1877.

[7] G. Scutari and D. P. Palomar, "MIMO cognitive radio: A game theoretical approach,” IEEE Trans. Sig. Proc., vol. 58, no. 2, pp. 761-780, 2010.

[8] S. Hua, H. Liu, M. Wu, and S. S. Panwar, "Exploiting MIMO antennas in cooperative cognitive radio networks," in Proc. IEEE INFOCOM, 2011, pp. 2714-2722.

[9] D. N. Nguyen and M. Krunz, "Spectrum management and power allocation in MIMO cognitive networks," in Proc. IEEE INFOCOM, 2012, pp. 2023-2031.

[10] V. R. Cadambe and S. Jafar, "Interference alignment and degrees of freedom of the K-user interference channel," IEEE Trans. Inform. Theory, vol. 54, no. 8, pp. 3425-3441, 2008.

[11] S. M. Perlaza, N. Fawaz, S. Lasaulce, and M. Debbah, "From spectrum pooling to space pooling: Opportunistic interference alignment in MIMO cognitive networks,” IEEE Trans. Sig. Proc., vol. 58, no. 7, pp. 3728-3741, 2010.

[12] C. Tsinos and K. Berberidis, "Blind opportunistic interference alignment in MIMO cognitive radio systems," IEEE J. Emerging and Sel. Topics in Circuits and Systems, vol. 3, no. 4, pp. 626-639, Dec 2013.

[13] L. Sboui, H. Ghazzai, Z. Rezki, and M.-S. Alouini, "Achievable rate of cognitive radio spectrum sharing MIMO channel with space alignment and interference temperature precoding,” in Proc. IEEE ICC’13, 2013, pp. 2656-2660.

[14] N. Zhao, T. Qu, H. Sun, A. Nallanathan, and H. Yin, "Frequency scheduling based interference alignment for cognitive radio networks," in Proc. IEEE GLOBECOM’13, Dec 2013, pp. 3447-3451.

[15] Y. Xu and S. Mao, "Stackelberg game for cognitive radio networks with MIMO and distributed interference alignment," IEEE Trans. Veh. Tech., vol. 63, no. 2, pp. 879-892, Feb 2014.

[16] M. El-Absi, M. Shaat, F. Bader, and T. Kaiser, "Interference alignment based resource management in MIMO cognitive radio systems," in Proc. Europ. Wirless. Conf.'14, May 2014, pp. 1-6.

[17] M. El-Absi and T. Kaiser, "Optimal resource allocation based on interference alignment for OFDM and FBMC MIMO cognitive radio systems," in Proc. Europ. Conference on Networks and Commun., June 2014, pp. 1-5.

[18] M. El-Absi, M. Shaat, F. Bader, and T. Kaiser, "Power loading and spectral efficiency comparison of MIMO OFDM/FBMC for interference alignment based cognitive radio systems,” in Proc. ISWCS'14, Aug 2014, pp. 480-485.

[19] N. Zhao, F. Yu, and H. Sun, "Adaptive energy-efficient power allocation in green interference alignment wireless networks," IEEE Trans. Veh. Tech., 2014.

[20] — - "Power allocation for interference alignment based cognitive radio networks," in Proc. IEEE INFOCOM WKSHPS'14, April 2014, pp. 742-746.

[21] S. Gollakota, S. Perli, and D. Katabi, “Interference alignment and cancellation,” in Proc. ACM SIGCOMM'09, ser. SIGCOMM '09, 2009, pp. 159-170. [Online]. Available: http://doi.acm.org/10.1145/1592568.1592588

[22] V. R. Cadambe and S. Jafar, "Reflections on interference alignment and the degrees of freedom of the K-user MIMO interference channel," in IEEE Inform. Theory Society Newsletter, 2009, pp. 5-8.

[23] S. Jafar and M. J. Fakhereddin, "Degrees of freedom for the MIMO interference channel,” IEEE Trans. Inform. Theory, vol. 53, no. 7, pp. 2637-2642, 2007.

[24] C. M. Yetis, T. Gou, S. Jafar, and A. H. Kayran, “On feasibility of interference alignment in MIMO interference networks,” IEEE Trans. Sig. Proc., vol. 58, no. 9, pp. 4771-4782, 2010.

[25] O. Gonzalez, I. Santamaria, and C. Beltran, "A general test to check the feasibility of linear interference alignment," in Proc. IEEE ISIT'12, 2012, pp. 2481-2485.

[26] R. Tresch, M. Guillaud, and E. Riegler, "On the achievability of interference alignment in the K-user constant MIMO interference channel,” in Proc. IEEE/SP Workshop on Statistical Signal Proc., Aug 2009, pp. 277-280.

[27] G. Bresler, D. Cartwright, and D. Tse, "Feasibility of interference alignment for the MIMO interference channel: The symmetric square case,” in Proc. IEEE ITW'11, 2011, pp. 447-451.

[28] K. Gomadam, V. R. Cadambe, and S. Jafar, "A distributed numerical approach to interference alignment and applications to wireless interference networks,” IEEE Trans. Inform. Theory, vol. 57, no. 6, pp. 3309-3322, 2011.

[29] Y. Xu and S. Mao, “On interference alignment in multi-user OFDM systems,” in Proc. IEEE GLOBECOM'12, Dec 2012, pp. 5339-5344.

[30] Y. Xu, S. Mao, and X. Su, "Interference alignment improves the capacity of OFDM systems,” IEEE Trans. Veh. Tech., 2015.

[31] G. Bansal, J. Hossain, and V. K. Bhargava, "Optimal and suboptimal power allocation schemes for OFDM-based cognitive radio systems,” IEEE Trans. Wireless Commun., vol. 7, no. 11, pp. 4710-4718, 2008.

[32] T. Weiss, J. Hillenbrand, A. Krohn, and F. K. Jondral, "Mutual interference in OFDM-based spectrum pooling systems," in Proc. IEEE 59th VTC'04, vol. 4, 2004, pp. 1873-1877.

[33] Q. Zhao, S. Geirhofer, L. Tong, and B. Sadler, “Opportunistic spectrum access via periodic channel sensing,” IEEE Trans. Sig. Proc., vol. 56, no. 2, pp. 785-796, Feb 2008. 
[34] T. Ban, W. Choi, B. Jung, and D. Sung, "Multi-user diversity in a spectrum sharing system,” IEEE Trans. Wireless Commun., vol. 8, no. 1, pp. 102-106, Jan 2009.

[35] S. Chen and R. Cheng, "Clustering for interference alignment in multiuser interference network," IEEE Trans. Veh. Tech., vol. PP, no. 99, pp. 1-1, 2013.

[36] S. Ben Halima and A. Saadani, "Joint clustering and interference alignment for overloaded femtocell networks," in IEEE Wireless Communications and Networking Conference (WCNC), 2012, pp. 1229-1233.

[37] P. Nikolaos and A. Theodore, "Bit and power allocation in constrained multicarrier systems: The single-user case,” EURASIP J. on Advances in Signal Proc., vol. 2008, 2007.

[38] W. Yu, W. Rhee, S. Boyd, and J. M. Cioffi, “Iterative water-filling for gaussian vector multiple-access channels,” IEEE Trans. Inform. Theory, vol. 50, no. 1, pp. 145-152, 2004.

[39] M. Pischella and E. Vivier, “Comparison of distributed space and frequency interference alignment," in Proc. IEEE PIMRC'10, Sept 2010, pp. 532-537.

[40] L. Ma, T. Xu, and G. Sternberg, “Computational complexity of interference alignment for symmetric MIMO networks,” IEEE Commun. Lett., vol. 17, no. 12, pp. 2308-2311, December 2013.

[41] C. Sun, Y. Yang, and Y. Yuan, "Low complexity interference alignment algorithms for desired signal power maximization problem of MIMO channels," EURASIP J. on Advances in Signal Proc., vol. 2012, no. 1, 2012. [Online]. Available: http://dx.doi.org/10.1186/1687-6180-2012-137

[42] S. W. Peters and R. W. Heath, "Interference alignment via alternating minimization,” in Proc. IEEE ICASSP'09, 2009, pp. 2445-2448.

[43] Y. J. Zhang and K. B. Letaief, "An efficient resource-allocation scheme for spatial multiuser access in MIMO/OFDM systems,” IEEE Trans. Commun., vol. 53, no. 1, pp. 107-116, Jan 2005.

[44] E. Lo, P. Chan, V. Lau, R. Cheng, K. B. Letaief, R. Murch, and W. Mow, “Adaptive resource allocation and capacity comparison of downlink multiuser MIMO-MC-CDMA and MIMO-OFDMA,” IEEE Trans. Wireless Commun., vol. 6, no. 3, pp. 1083-1093, March 2007.

[45] M. Grant and S. Boyd, "CVX: Matlab software for disciplined convex programming (web page and software)," in http://stanford.edu/ boyd/cvx, 2009. 